\title{
Coherent Synthesis of Terahertz Radiation from Femtosecond Laser Filaments in Air
}

\author{
Sergey I. Mitryukovskiy, Yi Liu, Bernard Prade, Aurélien Houard and André Mysyrowicz \\ Laboratoire d'Optique Appliquée, ENSTA ParisTech/CNRS/Ecole Polytechnique, 828, Boulevard des \\ Maréchaux, Palaiseau, F-91762, France
}

\begin{abstract}
Terahertz radiation from femtosecond laser filaments organized in an array. The Terahertz intensity is proportional to the square of the number of the filaments, which provides a simple method for scaling up of the Terahertz energy with powerful femtosecond laser. Moreover, directional off-axis terahertz radiation can be achieved. This paves the way for applications of this Terahertz source for remote sensing.
\end{abstract}

Electromagnetic radiation in the Terahertz $(\mathrm{THz})$ domain has received much attention in the last two decades. $\mathrm{THz}$ spectroscopy is ideally suited for the identification of biological or complex inorganic molecules [1], the study of collective excitations in condensed media [24], for plasma diagnostics [5]. Applications of $\mathrm{THz}$ radiation range from medicine [6], food industry process control [7], to security concerns [8], telecommunication [9], etc. Impressive progress has been achieved in the last few years in the development of intense $\mathrm{THz}$ sources. Optical rectification of a short laser pulse in a nonlinear crystal yields THz sources with field amplitude reaching the $\mathrm{MV} / \mathrm{cm}$ level [10]. Unfortunately, many applications which require illumination of a distant target are hampered by the poor transmission of $\mathrm{THz}$ radiation through atmosphere, due to the strong attenuation posed by water vapor.

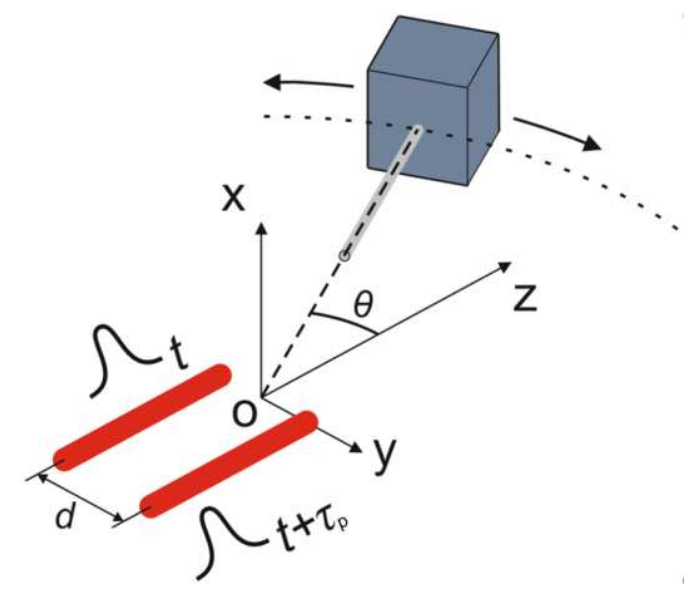

Fig. 1. Experimental setup. The two filaments are separated laterally by a variable distance of $d$. The adjustable temporal delay between the two filamentary pulses is $\tau_{\mathrm{p}}$

Recently, significant progress has been achieved in remote $\mathrm{THz}$ generation. To alleviate the strong attenuation in air, one produces a THz emitting source close to the distant target. This can be readily achieved using intense infrared femtosecond laser pulses. A first scheme consists in simply launching a short laser pulse in air. If the initial peak power exceeds a critical value $P_{c r} \equiv 3.72 \lambda_{0}^{2} / 8 \pi n_{0} n_{2}$, the pulse undergoes filamentation [11]. Here, $\lambda_{0}, n_{0}$ and $n_{2}$ are the laser wavelength, the refractive index of air and the nonlinear refractive index of air, respectively. The plasma column created during this process is initially in an excited state of longitudinal oscillations; it produces a forward oriented, radially polarized conical $\mathrm{THz}$ emission [12]. By simple manipulation of the initial laser beam, it is possible to place the ionized region, and hence the $\mathrm{THz}$ source, at a distance from the laser, which can reach 
hundreds of meters. This technique has poor energy conversion efficiency from IR to THz, on the order of $10^{-9}$ per filament [13]. However, the THz output can be increased by increasing the number of filaments. With a laser pulse of peak power $P>>P_{\mathrm{cr}}, N$ parallel filaments are formed, where $N \sim P / P_{\mathrm{cr}}$, adding their emission incoherently. For instance, a laser with terawatt power led to a $\mathrm{THz}$ radiation at $30 \mathrm{~m}$ from the laser with 40 times higher power than with a 12 gigawatt laser [12]. Note that there is no obvious limit to the further scaling up in laser power since there is no optical element susceptible of being damaged in the path of the laser.

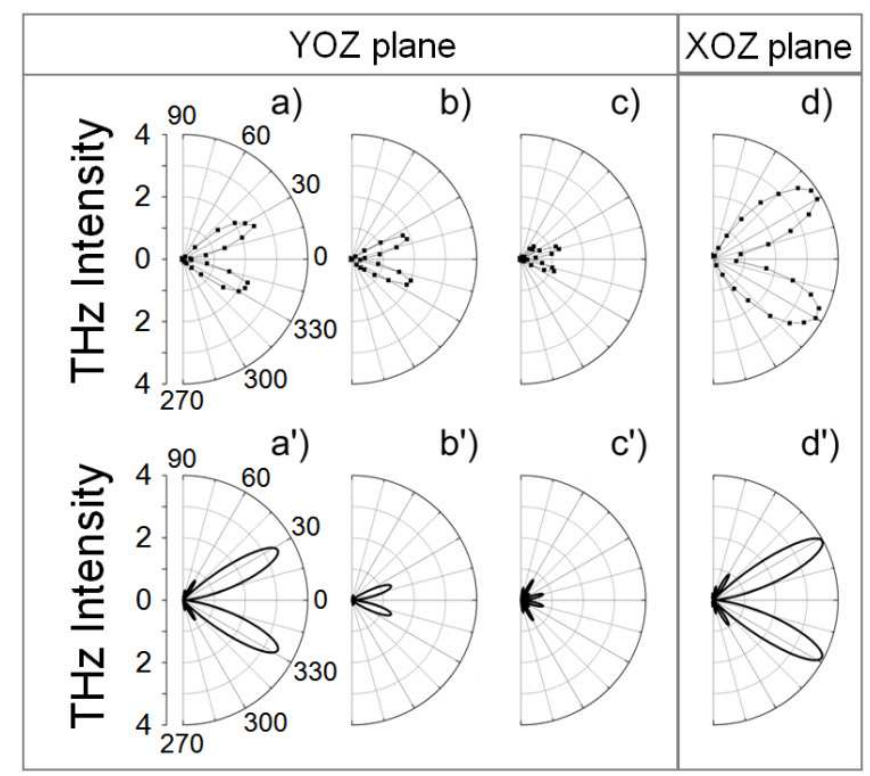

Fig. 2. (a)-(c) Emission diagrams of the $\mathrm{THz}$ radiation at $91 \mathrm{GHz}$ obtained in the YOZ plane from two filaments as a function of separation $d$. Top row: experimental results. Bottom row: calculations. From (a) to (c), the lateral separation $d$ is $0.5 \mathrm{~mm}, 2.4 \mathrm{~mm}$ and $3.4 \mathrm{~mm}$, respectively. (d) Emission diagram of the THz radiation obtained in the XOZ plane from two filaments laying in the YOZ plane and separated by $2.4 \mathrm{~mm}$. All THz intensities are normalized to the peak $\mathrm{THz}$ intensity of a single filament.

In a second scheme, the $800 \mathrm{~nm}$ femtosecond laser pulse is combined with its second harmonic at $400 \mathrm{~nm}$ for the generation of the plasma $\mathrm{THz}$ emitter. With the appropriate phase between fundamental and second harmonic fields, the plasma is created in an excited state with net transverse current [14]. For a short plasma string in the laboratory, the THz produced in this way was found to be $10^{2}-10^{3}$ times larger than with a single color pulse $[15,16]$. Recently two groups reported bicolor $\mathrm{THz}$ generation at a distance of $16-17 \mathrm{~m}$, with an enhancement by two orders of magnitude with respect to the single color case $[17,18]$. However, there are difficulties with the production of $\mathrm{THz}$ radiation by this method at longer distances [19]. The difficulties are tied to the process of filamentation, which appears unavoidably when $P>P_{\text {cr }}$ and generates an extended plasma column. In order to produce $\mathrm{THz}$ radiation efficiently, proper phase relation and temporal overlap between the $\omega$ and $2 \omega$ pulses must be maintained over the plasma length [19-21]. For instance, the THz emission produced by this two-color method at a distance of $55 \mathrm{~m}$ was only a small fraction of that at shorter distance, due to walk-off between fundamental and harmonic pulse [19].

In this letter, we demonstrate the coherent synthesis of the $\mathrm{THz}$ radiation from individual single color filaments organized in an array. We find that the THz intensity scales up with $N^{2}$ provided that proper filament separation and laser pulse time delays are chosen. Moreover, the $\mathrm{THz}$ radiation pattern can be controlled, which is a useful property for many applications. Calculations based on the Transition-Cherenkov model of $\mathrm{THz}$ emission by filaments agree well with our observations.

The $\mathrm{THz}$ field distribution in the far field from a single filament reads [22] 


$$
E_{\theta}=\frac{1}{4 \pi} \sqrt{\frac{\mu_{0}}{\varepsilon_{0}}} j(\omega) \frac{e^{i k R}}{R} \sin \theta \frac{e^{i k L(1-\cos \theta)}-1}{1-\cos \theta}
$$

where $\omega, L, \theta, k$ represent the frequency of the considered $\mathrm{THz}$ component, the length of the filament, the angle of the radiation and the $\mathrm{THz}$ wave number, respectively. The spectral current amplitude at frequency $\omega$ is given by

$$
j(\omega)=\varepsilon_{0} E_{L} \omega_{p e} \frac{\omega+2 i v_{e}}{\omega_{p e}^{2}-\omega^{2}+i v_{e} \omega} \frac{\sin \left(\omega \tau_{L} / 2\right)}{1-\left(\omega \tau_{L} / 2 \pi\right)^{2}} \exp \left(-\frac{i \omega \tau_{L}}{2}\right),
$$

where $E_{L}=e \omega_{p e} I_{0} / 2 m_{e} \varepsilon_{0} c^{2} \omega_{0}^{2}$ is the amplitude of the plasma wave, $\omega_{p e}=\sqrt{e^{2} n_{e} / m_{e} \varepsilon_{0}}$ is the electron plasma frequency, $\omega_{0}$ is the laser circular frequency, $v_{e}$ is the electron collision frequency, and $\tau_{L}$ is the laser pulse duration. The model can be readily extended to the case of $N$ identical filaments. The interference of these THz fields yields

$$
E_{\theta}^{\text {total }}=\frac{1}{4 \pi} \sqrt{\frac{\mu_{0}}{\varepsilon_{0}}} j(\omega) \sum_{i=1}^{N} e^{i \phi_{i}} \frac{e^{i k R_{i}}}{R_{i}} \sin \theta_{i} \frac{e^{i k L\left(1-\cos \theta_{i}\right)}-1}{1-\cos \theta_{i}} .
$$

Here $\phi_{i}=\omega \tau_{i}$ is the accumulated phase difference of the THz fields due to the temporal delay $\tau_{\mathrm{i}}$ between the laser pulses and $R_{i}$ is the distance from the $i^{\text {th }}$ filament emitter to the detector.

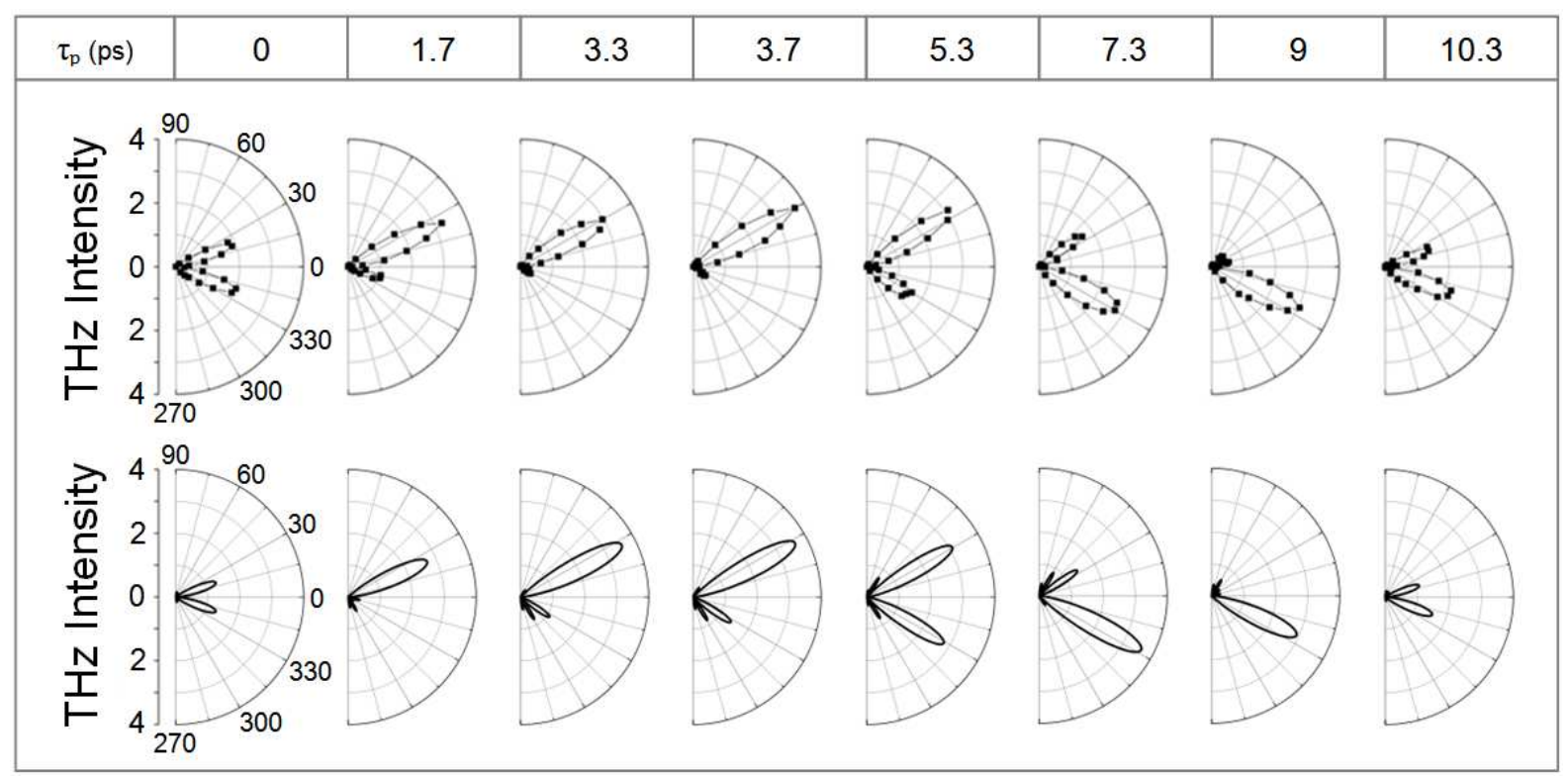

Fig. 3. Evolution of emission diagrams of the THz radiation in the YOZ plane from two filaments as a function of the temporal delay between the two laser pulses. The two filaments are separated by $d=2.4 \mathrm{~mm}$. Top row: experimental results. Bottom row: calculations.

To verify the model, two IR femtosecond laser pulses of 40 fs duration, with equal energy of $1.1 \mathrm{~mJ}$, obtained from a Mach-Zehnder interferometer were focused by two convex lenses $(f=100 \mathrm{~cm})$ to form two parallel filaments in ambient air. The experimental setup is presented in Fig. 1. The spatial separation and temporal delay of the two pulses could be controlled by a steering mirror of the interferometer and an integrated mechanical delay line. The forward $\mathrm{THz}$ radiation was detected by a heterodyne detector working at $91 \mathrm{GHz}$ [23]. 
For the two parallel filaments in the horizontal plane, the detector was rotated in the YOZ and $\mathrm{XOZ}$ plane. The synthesized $\mathrm{THz}$ field intensity emitted in the YOZ plane is presented in Figs. 2(a)-(c) for different spatial separations and zero temporal delay $\left(\tau_{\mathrm{p}}=0\right)$. With the increase of the separation $d$ from $0.5 \mathrm{~mm}$ to $3.4 \mathrm{~mm}$, the $\mathrm{THz}$ radiation diminishes but remains symmetric. The emission in the XOZ plane for $d=2.4 \mathrm{~mm}$ is shown in Fig. 2(d). Results as a function of time delay for a separation $d=2.4 \mathrm{~mm}$ are shown in Fig. 3 (top row). Upon increase of the delay $\tau_{\mathrm{p}}$, at fixed separation, the radiation pattern becomes asymmetric. With $\tau_{\mathrm{p}}=3.7 \mathrm{ps}$, the radiation is totally directed along one lobe. The peak THz intensity is observed to be 3.8 times larger than that of a single filament. With further delay, after $\tau_{\mathrm{p}}=$ $\lambda / 2 \mathrm{c}=5.5 \mathrm{ps}$, another lobe starts to grow at the expense of the previous one. For $\tau_{\mathrm{p}}=10.3 \mathrm{ps}$ $(\sim \lambda / \mathrm{c})$, the radiation pattern becomes nearly identical to that without time delay $\left(\tau_{\mathrm{p}}=0\right)$.

Figures 2 and 3 (bottom row) show the calculated $\mathrm{THz}$ radiation patterns for the corresponding separations and delays. Excellent agreement between the experimental results and calculation is found. The emission pattern at other $\mathrm{THz}$ frequencies can be obtained from equations (2) and (3).

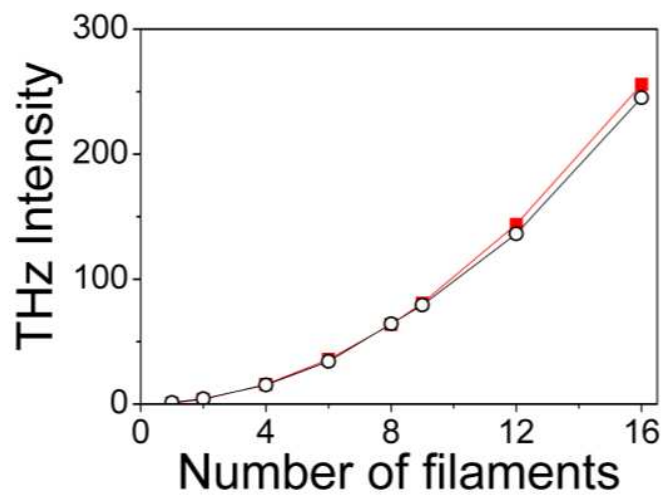

Fig. 4. Dependence of peak $\mathrm{THz}$ intensity on the number of filaments $N$. Squares - theoretical upper limit (quadratic dependence), Circles - maximum value obtained in our calculations.

The good agreement between our experimental observations and calculations validates the theoretical model and permits to extrapolate the results to a higher number of filaments. It has been demonstrated that a large number of filaments can be organized in regular patterns by manipulation of the beam profile of a femtosecond laser pulse with $P>>P_{\text {cr }}$ [24-26]. We have calculated the THz radiation distribution for $N=4,6,8,9,12,16$ filaments organized in a square grid. With certain combinations of filament delays, quite complicated radiation patterns can come up. With a properly optimized delay, the total $\mathrm{THz}$ energy can be channeled towards a preferential direction. The corresponding peak intensity scales almost like $N^{2}$, as shown in Fig. 4. In Fig. 5, we present the normalized three dimensional distribution of the optimized $\mathrm{THz}$ field for 1,2 and 16 filaments separated by $d=2.4 \mathrm{~mm}$. With two filaments and zero delay, a butterfly wing pattern appears in the XOZ plane (Fig. 5 (b)). Figure $2\left(b^{\prime}\right)$ and $\left(d^{\prime}\right)$ are actually cross section intensity distributions of this radiation pattern. A single leaf radiation pattern is obtained with $\tau_{\mathrm{p}}=3.5 \mathrm{ps}$ (Fig. 5(c)). This can prove interesting in applications where irradiation of targets with intense laser pulse should be avoided. For a larger number of filaments, similar leaf-shape radiation pattern appear with properly chosen pulse delays. For example, the synthesized $\mathrm{THz}$ radiation from 16 filaments is presented in Fig. 5(d), where its peak intensity is found to be $\sim 250$ times that of a single filament. Recently, M. Châteauneuf and coworkers have reported the generation of $10^{3}$ filaments organized in a circle [26]. With proper phase control, it could lead to a $10^{6}$ increase of THz peak intensity. 
There is a large number of possible combinations for the interference of $\mathrm{THz}$ fields from individual filaments. For instance, we have studied experimentally and theoretically the emission of non parallel filaments, forming the equivalent of a vee antenna [27]. We expect to obtain circularly polarized $\mathrm{THz}$ field if proper delays between the filament antennas are chosen. We have also verified that it is possible to combine coherently the output from two bicolor filaments. A highly directional central $\mathrm{THz}$ radiation is obtained in both cases.

a)

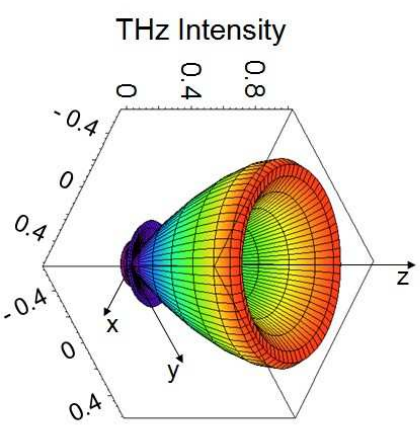

b)

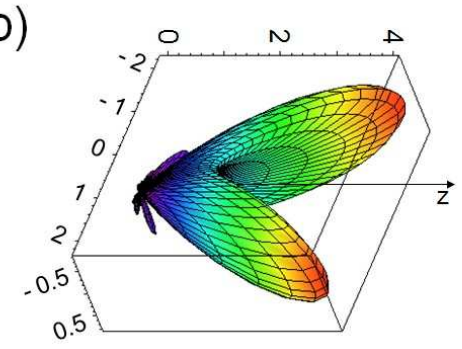

C)

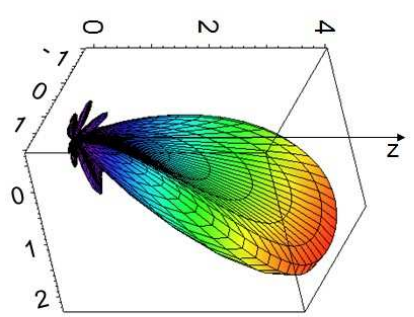

d)

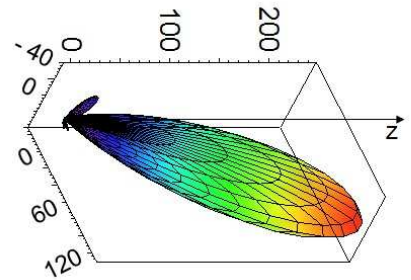

Fig. 5. (a) Calculated $\mathrm{THz}$ radiation diagram from single filament. Calculated $\mathrm{THz}$ radiation diagram from two parallel filaments separated by $2.4 \mathrm{~mm}$ : (b) without time delay, (c) with a time delay $\tau_{\mathrm{p}}=3.5 \mathrm{ps}$. (d) Calculated $\mathrm{THz}$ radiation diagram from 16 filaments with proper time delays.

In conclusion, we have discussed the coherent synthesis of the $\mathrm{THz}$ radiation from an array of $N$ femtosecond laser filaments. This provides a simple technique for scaling up the $\mathrm{THz}$ intensity with high power femtosecond laser since the total $\mathrm{THz}$ intensity is proportional to $N^{2}$. With appropriate delay between the filaments, the emission can be directed along preferential directions, which is of high interest for remote $\mathrm{THz}$ applications. 


\section{References:}

[1] H. Zhong, A. Redo-Sanchez, and X.-C. Zhang, Opt. Express, 14, 9130 (2006).

[2] T. Suzuki and R. Shimano, Phys. Rev. Lett. 109, 046402 (2012).

[3] R. Matsunaga and R. Shimano, Phys. Rev. Lett. 109, 187002 (2012).

[4] T. Kampfrath, et al., Nature Photon. 5, 31 (2011).

[5] S. P. Jamison, et al., J. Appl. Phys. 93, 4334 (2003).

[6] A. J. Fitzgerald, et al., Phys. Med. Biol. 47, 67 (2002).

[7] Y. K. Lee, et al., J. Food Prot. 75, 179 (2012).

[8] H.-B. Liu, et al., Proc. IEEE 95, 1514 (2007).

[9] J. Federici and L. Moeller, J. Appl. Phys. 107, 111101 (2010).

[10] H. Hirori, et al., Appl. Phys. Lett. 98, 091106 (2011).

[11] A. Couairon and A. Mysyrowicz, Phys. Rep. 441, 47 (2007).

[12] C. D'Amico, et al., Phys. Rev. Lett. 98, 235002 (2007).

[13] A. Houard, et al., Appl. Phys. Lett. 91, 241105 (2007).

[14] K. Y. Kim, et al., Opt. Express, 15, 4577 (2007).

[15] T. Löffler et al., Semicond. Sci. Technol. 20, 134 (2005).

[16] X. Xie, J. Dai, and X.-C. Zhang, Phys. Rev. Lett. 96, 075005 (2006).

[17] T.-J. Wang, et al., Appl. Phys. Lett. 97, 111108 (2010).

[18] J. Dai, et al., IEEE J. Sel. Top. Quant. Electron. 17, 183 (2011).

[19] J.-F. Daigle, et al., Opt. Express, 20, 6825 (2012).

[20] Y. Liu, et al., Opt. Express, 17, 11480 (2009).

[21] Y. S. You, T. I. Oh, and K. Y. Kim, Phys. Rev. Lett. 109, 183902 (2012).

[22] C. D'Amico, et al., New J. Phys. 10, 013015 (2008).

[23] S. Tzortzakis, et al., Opt. Lett. 27, 1944 (2002).

[24] G. Méchain, et al., Phys. Rev. Lett. 93, 035003 (2004).

[25] H. Schroeder, J. Liu, and S. Chin, Opt. Express, 12, 4768 (2004).

[26] M. Châteauneuf, et al., Appl. Phys. Lett. 92, 091104 (2008).

[27] K. A. Balanis, Antenna Theory: Analysis and Design, Third edition, John Wiley \& Sons, Inc. (Hoboken, New Jersey, 2005). 